\title{
QUEEN'S
UNIVERSITY
BELFAST
}

\section{Quantifying protein interaction dynamics by SWATH mass spectrometry: application to the 14-3-3 system}

Collins, B. C., Gillet, L. C., Rosenberger, G., Röst, H. L., Vichalkovski, A., Gstaiger, M., \& Aebersold, R. (2013). Quantifying protein interaction dynamics by SWATH mass spectrometry: application to the 14-3-3 system. Nature Methods, 10(12), 1246-1253. https://doi.org/10.1038/nmeth.2703

Published in:

Nature Methods

Document Version:

Peer reviewed version

Queen's University Belfast - Research Portal:

Link to publication record in Queen's University Belfast Research Portal

Publisher rights

(c) 2013 Nature America, Inc. All rights reserved. This work is made available online in accordance with the publisher's policies. Please refer to any applicable terms of use of the publisher.

\section{General rights}

Copyright for the publications made accessible via the Queen's University Belfast Research Portal is retained by the author(s) and / or other copyright owners and it is a condition of accessing these publications that users recognise and abide by the legal requirements associated with these rights.

Take down policy

The Research Portal is Queen's institutional repository that provides access to Queen's research output. Every effort has been made to ensure that content in the Research Portal does not infringe any person's rights, or applicable UK laws. If you discover content in the Research Portal that you believe breaches copyright or violates any law, please contact openaccess@qub.ac.uk. 


\section{Quantifying protein interaction dynamics by SWATH mass spectrometry: application to the $14-3-3$ system}

Ben C. Collins ${ }^{1}$, Ludovic C. Gillet ${ }^{1}$, George Rosenberger ${ }^{1,2}$, Hannes L. Röst ${ }^{1,2}$, Anton Vichalkovski ${ }^{1}$, Matthias Gstaiger ${ }^{1}$, and Ruedi Aebersold 13,4

1Department of Biology, Institute of Molecular Systems Biology, ETH Zurich, 8093 Zurich, Switzerland

${ }^{2}$ Ph.D. Program in Systems Biology, University of Zurich and ETH Zurich, CH-8057 Zurich, Switzerland

${ }^{3}$ Competence Center for Systems Physiology and Metabolic Diseases, 8093 Zurich, Switzerland

${ }^{4}$ Faculty of Science, University of Zurich, 8057 Zurich, Switzerland

Correspondence should be addressed to R. A. (aebersold@imsb.biol.ethz.ch)

\section{Editorial Summary Dynamic changes to the 14-3-3 protein interactome are robustly followed over time using affinity purification-data independent analysis-based mass spectrometry. Also online, Lambert et al. describe a similar method.}




\begin{abstract}
Protein complexes and protein interaction networks are essential mediators of most biological functions. Complexes supporting transient functions such as signal transduction processes are frequently subject to dynamic remodeling. Currently, the majority of studies into the composition of protein complexes are carried out by affinity purification and mass spectrometry (AP-MS) and present a static view of the system. To better understand inherently dynamic biological processes, methods to reliably quantify temporal changes of protein interaction networks are essential. Here, we used affinity purification combined with SWATH mass spectrometry (AP-SWATH) to study the dynamics of the 14-3-3 $\beta$ scaffold protein interactome after stimulation of the insulin-PI3K-AKT pathway. The consistent and reproducible quantification of 1,967 proteins across all stimulation time points provided insights into the 143-3 $\beta$ interactome and its dynamic changes following IGF1 stimulation. We therefore establish AP-SWATH as a tool to quantify dynamic changes in protein complex interaction networks.
\end{abstract}

\title{
Introduction
}

Cellular functions are rarely attributed solely to a single molecule. Typically, they are carried out by sets of molecules organized into functional modules ${ }^{1}$. This is especially true in the case of proteins associating to form functional multi-subunit protein complexes ${ }^{2}$. These range from well-defined molecular machines to more dynamic structures including transient interactions typical in signaling pathways. Scaffold, adaptor, or anchor proteins (generalized as scaffold proteins) are of particular interest in signaling as they can mediate the spatial localization of components in a cascade and take a central role in cellular processes as hubs controlling the flow of information ${ }^{3}$. In a growing number of signaling systems, scaffold proteins are thought to have essential functions such as facilitating assembly of functional protein complexes, causing allosteric changes in ligand proteins, or affecting subcellular localization ${ }^{4}$. This apparently central role of scaffold proteins makes them high value targets in protein interaction studies, particularly for time-resolved measurements in perturbed systems where the reorganization of protein complexes is common.

14-3-3 proteins are a family of 7 abundant cellular scaffolds which form homo- and heterodimers with diverse regulatory functions in eukaryotes ${ }^{5}$. A 14-3-3 dimer can bind 2 phosphorylated serine or threonine residues situated on the same or different ligand proteins. The phosphosites recognized by 14-3-3 proteins are in a sequence motif including an arginine in the -3 or -4 position and a hydrophobic residue in the +2 position. The 14-3-3 ligand motif therefore facilitates direct physical interactions but in many cases the ligand itself is complexed to other proteins, and represents the attachment point to a multi-subunit complex. Therefore, in APs of 14-3-3 proteins we expect to co-purify proteins that represent indirect 14-3-3 interactions. 14-3-3 proteins are thought to be among the most connected in the proteome with respect to protein-protein interactions, with several hundred interactions reported per isoform 5 (previous 14-3-3 interaction studies ${ }^{6-9}$ are described in Supplementary Note 1). Phosphorylation of 14-3-3 ligand proteins is associated with activity of basophilic kinases, which have consensus substrate motifs related to the 14-3-3 ligand motif. Insulin-IGF1 signaling has been shown to be directly linked with the 14-3-3 system, and several AKT kinase substrates are known to bind 14-3-35, as shown in studies which compared the proteins interacting with 14-33 in on and off states of this pathway ${ }^{10-12}$. 
AP-MS (affinity purification - mass spectrometry) represents the method of choice to chart protein-protein interactions under near physiological conditions ${ }^{13,14}$. Proteome-wide interaction maps have been produced in model systems ${ }^{15-17}$, and many human protein interaction modules have been described with great detail18-20 and robustness ${ }^{21}$. While extensive information on protein-protein connectivity is a valuable asset, results from these studies describe a static representation of interaction networks only. There is an increasing awareness that to move toward a more complete understanding of inherently dynamic biological processes it is essential to generate time-resolved, quantitative descriptions of protein interaction networks in perturbed states ${ }^{22}$. Until now this problem has been approached chiefly using MS1 intensitybased quantification from AP-MS analysis of perturbed cell systems ${ }^{10,23-26}$. Such studies have enjoyed a good degree of success, but remain hampered due to the technical limitations of quantitative MS1 intensity-based and shotgun proteomics approaches ${ }^{27}$ relating to data completeness, linear dynamic range, and sensitivity. As such, the number of AP-MS studies which have attempted to detect changes in protein-protein interaction networks in perturbed systems remains small, at least with respect to projects engaged in large scale interaction mapping13,14,22. A recent development has seen the application of targeted proteomics via SRM (selected reaction monitoring) to differential interaction proteomics in an attempt to address some of these limitations ${ }^{28,29}$. Termed AP-SRM, this approach succeeded in providing quantitatively complete and sensitive measurements of a perturbed scaffold-centric protein interaction network (see Supplementary Note 1).

Classical shotgun proteomics relies on DDA (data dependent acquisition), i.e. selection in real time of precursors for fragmentation. As DDA is a semi-stochastic process the set of peptides identified across samples is not reproducible as long as the number of detected precursors exceeds the number of available sequencing cycles ${ }^{27}$. This is in contrast to SRM which reproducibly targets a predetermined peptide set for quantification. DIA (data independent acquisition) collects MS2 spectra for the entire expected mass range of tryptic peptides by coisolating and fragmenting multiple peptide precursors in isolation windows ranging from a few $\mathrm{m} / \mathrm{z}$ to the entire mass range simultaneously ${ }^{30-34}$. SWATH-MS ${ }^{35}$ is a recently described implementation of DIA which cycles through fixed precursor isolation windows (e.g. $25 \mathrm{~m} / \mathrm{z}$ ) using a quadrupole-time-of-flight mass spectrometer achieving essentially complete peptide fragment ion coverage for precursors in the tryptic peptides mass range. An essential feature that distinguishes SWATH-MS from other DIA strategies is the use of prior knowledge regarding fragmentation and chromatographic behavior of target peptides. This information is used for scoring signal groups extracted from SWATH-MS datasets to identify and quantify peptides automatically and at large scale ${ }^{36}$ (unpublished data H. R., G. R.; www.openswath.org). SWATHMS provides SRM-like performance in terms of quantitative accuracy, data completeness and dynamic range without specifying target peptides prior to data acquisition. Further, and unlike SRM, SWATH-MS can quantify an unlimited number of target peptides as long as they have been previously observed by shotgun MS.

We speculated that SWATH-MS could provide solutions to issues impeding progress in quantitative interaction proteomics. To assess the capability of AP combined with SWATH-MS (AP-SWATH) to rapidly and reliably identify and consistently quantify protein-protein interactions in time-resolved perturbation experiments, we chose to study the dynamics of 14-3$3 \beta$ scaffold protein interactome after stimulation of the insulin-PI3K-AKT pathway. 


\section{Results}

Characterization of the 14-3-3 $\beta$ signaling system

We selected 14-3-3 as a challenging system in which to evaluate the capability of AP-SWATH to consistently quantify large numbers of protein-protein interactions across multiple conditions and generated a stable HEK293 cell line inducibly expressing SH tagged $14-3-3 \beta$ as described ${ }^{18}$

(Supplementary Fig. 1). We chose IGF1 stimulation to perturb the 14-3-3 interactome because prior data showed that substrates of AKT kinase, a central mediator of insulin-IGF1 signaling, frequently bind 14-3-3 proteins ${ }^{5}$. As serum starvation was not sufficient to abolish AKT activation, we included a 60 minute pretreatment with the reversible PI3K inhibitor LY294002 to achieve a PI3K inactive ground state. The time course consisted of 6 conditions in biological triplicate (no treatment, - $60 \mathrm{~min}$; after LY294002 pre-treatment, $0 \mathrm{~min}$; and 4 time points after IGF1 stimulation, $1 \mathrm{~min}, 10 \mathrm{~min}$; $30 \mathrm{~min}$, and $100 \mathrm{~min}$ - see Fig. 1a). We confirmed activation of the insulin-IGF1 pathway by Western blotting for an activating phosphorylation on AKT (Supplementary Fig. 1a). Having established the 14-3-3 $\beta$ expression system, in which the bait was expressed 3-4 times below the endogenous level (Supplementary Fig. 1b), and confirmed that the stimulation was effective, we performed APs of 14-3-3 $\beta$ from the time course. We characterized these samples by shotgun mass spectrometry leading to the identification of 31,509 unique peptide sequences (FDR $0.2 \%$ ) corresponding to 2,532 unique proteins (FDR $0.3 \%$ ) across the entire experiment. These data served as the basis for generating reference spectra to quantify target peptides from AP-SWATH datasets.

\section{Generation and analysis of AP-SWATH maps}

The 18 affinity purified 14-3-3 $\beta$ samples from 6 conditions in the time course (Fig. 1), plus 9 GFP control APs, were subjected to SWATH-MS (Online Methods). The targeted data analysis paradigm employed to identify and quantify peptides from SWATH-MS data is based on prior knowledge of the fragmentation and chromatographic properties of each peptide. We used the shotgun MS data to construct a consensus peptide MS/MS spectral library37 (Fig. 1b) containing 31,469 sequence unique peptides and 41,934 MS2 consensus spectra including charge state and modifications. We selected the 5 most intense fragments for each peptide precursor ion resulting in 37,867 target assays containing 189,335 fragment ions for extraction of the APSWATH dataset (Supplementary Table 1).

We analysed the AP-SWATH data using OpenSWATH, an open source software for automated targeted analysis of DIA datasets (unpublished data H. R., G. R.; www.openswath.org). The targeted analysis workflow consists of extracting ion chromatograms for groups of fragment ions from a given peptide precursor in the appropriate MS2 SWATH map, and scoring peak groups detected in these chromatograms with respect to prior knowledge from peptide MS2 spectral libraries (Supplementary Fig. 2 and 3 and Online Methods). From the 14-3-3 APSWATH dataset we identified 19,123 peptide features (corresponding to 1,967 proteins) at 1\% FDR in 3 out of 3 biological replicates from at least one experimental condition (Supplementary Fig. 4). The result was an essentially complete quantitative data matrix, displayed as a heat map (Fig. 2 - source data is provided in the supplementary material), for all 1,967 proteins in all conditions (see Supplementary Results for information on quantitative reproducibility). This data matrix formed the basis for downstream quantitative comparisons relating firstly to filtering of non-specific contaminants and identifying high confidence 14-3-3 $\beta$ interactions, and secondly for examining the dynamics of $14-3-3 \beta$ interacting proteins over the perturbation. 


\section{AP-SWATH identifies 500+ high confidence 14-3-3ß interactions}

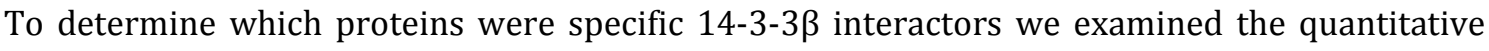
AP-SWATH data for enrichment of proteins in 14-3-3 $\beta$ APs compared with GFP. To determine statistical significance of $14-3-3 \beta$ versus GFP controls we calculated protein fold changes and adjusted p-values ${ }^{38}$. A volcano plot (Fig. 3a) indicates very strong enrichment of proteins in 143-3 $\beta$ purifications compared to control purifications averaged over all conditions. The corresponding plot area for GFP purifications is essentially devoid of proteins other than the control bait itself (GFP), 2 zinc finger proteins (Q9H5H4 and 060290) and another DNA binding protein (Q15424) which are very consistently enriched in control purifications for unknown reasons. We filtered the dataset at a significance level of adjusted p-value $<0.01$, and $\log 2 \mathrm{FC}>2$ to produce a high confidence 14-3-3 $\beta$ interactome containing 567 proteins (Supplementary Table 2). To assess the validity of $14-3-3 \beta$ protein interactions identified by AP-SWATH, we performed reciprocal purifications on a subset of proteins. We selected 21 protein kinases spanning the majority of the abundance range (Supplementary Table 3), isolated the respective complexes by AP and analyzed the samples by shotgun MS. We found 14-3-3 $\beta$ as an interactor in 19 out of 21 kinases, whereas, no 14-3-3 $\beta$ was detected in APs from GFP or from 21 additional kinases selected as negative controls (Fig. 3b). The 2 kinases (PLK1 and PI3R4) not verified by reciprocal purifications demonstrated the highest abundance in 14-3-3 $\beta$ APs from IGF1 stimulated cells, whereas reciprocal experiments did not include stimulated conditions. As such, these 2 kinases still likely represent true protein interactions only apparent in stimulated cells.

As direct interaction partners of 14-3-3 proteins generally contain a 14-3-3 ligand motif, we determined if it was overrepresented in the 14-3-3 $\beta$ interactome set. We calculated the sliding fraction of 14-3-3 ligand motifs contained in all proteins identified by AP-SWATH ranked by fold change over control APs (Supplementary Figure 5). Approximately $1 / 3$ of the 567 high confidence 14-3-3 $\beta$ interacting proteins contained the motif, compared to less than $1 / 10$ of the SwissProt human proteome, indicating very substantial enrichment ( $\mathrm{p}$-value $=1.3 \mathrm{E}-59$, hypergeometric test - see Supplementary Results for gene ontology analysis). Enrichment of 14-3-3 ligand motifs did not return to the baseline value until protein $\sim 800-900$ in the rank, suggesting there are $\sim 20-30 \%$ additional $14-3-3 \beta$ interacting proteins which did not satisfy

statistical significance, and could not be called as high confidence interactions. To our knowledge, this study represents the largest reported interactome for a single bait, and further indicates that at least $2.8 \%$ of the proteome can be engaged by 14-3-3 dimers containing the 14$3-3 \beta$ isoform.

Having used the AP-SWATH quantitative data to identify 567 high confidence 14-3-3 $\beta$ interacting proteins, we went on to characterize their dynamics. We made a subset of the quantitative matrix (Fig. 2) containing 14-3-3 $\beta$ protein interactions and proceeded with the time series analysis.

\section{Time-resolved quantification of a perturbed interaction network}

The completeness of the measurements enabled facile calculation of statistics ${ }^{38}$ to determine the remodeling of the 14-3-3 $\beta$ interactome after IGF1 stimulation (see Supplementary Table 4). 
The temporal profiles of 14-3-3 $\beta$ binding to 6 selected representative proteins are shown (Fig. 4a-f). To detect if consistent patterns existed in the temporal profiles of 14-3-3 $\beta$ interacting proteins we applied fuzzy c-means clustering. 220 proteins which passed a non-strict filter for differential binding were assigned to any of the 5 distinct clusters (Fig. 4g-k and Supplementary Table 5). The first 5 line plots (Fig. 4a-e) are prototypical examples of proteins from the 5 clusters found (Fig. 4g-k respectively, see Supplementary Fig 6 for all line plots). To further verify these MS derived quantitative patterns we performed Western blotting on 6 selected 14-3-3 $\beta$ interactors. The quantitative profiles detected by MS and Western blotting were generally in very good agreement (Supplementary Figure 7).

AKTS1 (Fig. 4a) is an inhibitor of mTORC1 activity, canonical AKT substrate and 14-3-3 ligand protein ${ }^{39}$. It is thought that phosphorylation of AKTS1 by AKT induces 14-3-3 binding of AKTS1 leading to dissociation of AKTS1 from mTORC1 and subsequent reduction of mTORC1 inhibition. Consistent with this, we found a dramatic reduction of AKTS1 binding to 14-3-3 $\beta$ after treatment with a PI3K-AKT inhibitor and an immediate and sustained regain of binding after 1 minute of treatment with IGF1. Likewise, MYCBP2 (Fig. 4b), an E3 ubiquitin ligase and predicted substrate $^{40}$ of AKT, showed a similar temporal profile, somewhat delayed, with the largest increase in 14-3-3 $\beta$ binding occurring after 10 minutes of IGF1 treatment. Clusters 1 and 2 (containing AKTS1 and MYCBP2, respectively) have a similar profile except that cluster 1 demonstrates a very fast response to IGF1 treatment with the biggest changes occurring after 1 minute, and cluster 2 shows slower response with the largest increases after 10 minutes. We checked the enrichment of known and predicted AKT substrates over the background proteome in each of the 5 clusters (Fig. 4i). While all clusters were significantly enriched, clusters 1 and 2 had the greatest overrepresentation of AKT substrates (see Supplementary Results for description of clusters 3-5).

Given the dynamics of AKTS1, we chose to examine the behavior of other mTOR complex components ${ }^{41}$ present as $14-3-3 \beta$ interactions. We retrieved the subset of 14-3-3 $\beta$ interacting proteins directly connected to mTOR kinase by previously described literature interactions (Fig. 5a). In addition to mTOR kinase, these include the mTORC1 component AKTS1, and the mTORC2 components SIN1, PRR5, and RICTR (some peptides for RPTOR and LST8 were also detected, however, the abundance was $\sim 10-30$ times lower than for other mTORC components and did not have enrichment patterns consistent with specific 14-3-3 $\beta$ interactions). We extracted the quantitative profiles of these proteins over the perturbation (Fig. 5b). As described above, AKTS1 binding to 14-3-3 $\beta$ changes dramatically over the time course, in contrast to the mTORC2 specific subunits which display essentially no change. Binding of AKTS1 to 14-3-3 proteins is known to be regulated via T246 phosphorylation by AKT ${ }^{39}$. The AKTS1 phosphopeptide corresponding to this site was detected by AP-SWATH (Fig. 5c - see Online Methods regarding phosphopeptide extraction from AP-SWATH), and the time course pattern of this phosphopeptide corresponded faithfully to that of AKTS1 (Fig. 5d). The phosphopeptide supporting the previously reported direct RCTOR-14-3-3 $\beta$ interaction ${ }^{42}$ was not detected. We created a schematic representation of the mTORC complexes as described in the literature $39,41,42$, overlaid with information from the AP-SWATH data in unstimulated and IGF1 stimulated states (Fig. 5e). Here, we show mTORC2 as essentially unchanged with respect to the stimulation, while AKTS1 binding to $14-3-3 \beta$ is dramatically increased, consistent with the known model of AKTS1 phosphorylation and sequestration to 14-3-3 leading to relief of mTORC1 inhibition 39 . This, combined with the absence of mTORC1 specific subunits and radically different time course profiles of AKTS1 and mTOR suggests that the bulk of mTOR kinase in the 14-3-3 $\beta$ APs 
originates from the cellular mTORC2 pool and not mTORC1. This observation is based on previous literature knowledge ${ }^{39}$ in addition to AP-SWATH data, however, we would emphasize that such literature corroborative conclusions could not have been made using static AP-MS maps. The dynamic data serves to highlight the separate behaviors of mTORC1 and mTORC2 leading to suggestions of how protein complexes are reorganized (Fig. 5e), as opposed to a static protein interaction network representation (Fig. 5a). Overall, quantitative analysis via APSWATH provides a deep representation of the dynamic reorganization in the 14-3-3 $\beta$ interactome in perturbed states.

\section{Dynamic range of 14-3-3 $\beta$ interactome}

Given the large number of 14-3-3 $\beta$ interacting proteins detected, we elected to estimate the range of their abundances via the 'best flyer peptide' approach ${ }^{43}$. As SWATH-MS data processed via targeted extraction has the same structure as SRM data, in addition to being very highly correlated quantitatively with $\mathrm{SRM}^{35,44}$, we used an implementation of this strategy recently validated for SRM ${ }^{45}$. We plotted the estimated $\log 10$ protein abundances for the 14-3-3 $\beta$ interacting proteins ordered from high to low abundance (Fig. 6., data in Supplementary Table 6). A striking feature of this visualization is the wide dynamic range of abundance, more than 4 orders, over which the 14-3-3 $\beta$ interacting proteins are spread. In other words, for every copy of the least abundant ligand protein we detect in complex with $14-3-3 \beta$, there are $\sim 10,000$ copies of the most abundant ligand protein-14-3-3 complex.

We queried protein interaction databases to retrieve previously detected $14-3-3 \beta$ interactions. Our dataset covered $43.3 \%$ of 231 literature interactions for 14-3-3 $\beta$ (i.e. 99 proteins of which

51 contained the motif). We additionally compared our results against 290 'gold standard' direct 14-3-3 interacting proteins in the literature for which a phosphorylation 14-3-3 motif was identified 9 . We found $36.9 \%$ of these proteins, which were determined to bind any of 14-3-3 isoforms in a wide variety of samples, were detected in our data. While in our study the abundance of the interacting proteins containing the 14-3-3 binding motif spanned 4+ order of magnitude (Fig. 6), the matched 14-3-3 $\beta$ literature interactions showed a clear bias towards the upper 2.5 orders of abundance. This analysis suggests that our method is extremely sensitive in detecting interactions, particularly in highly complex scaffold protein APs.

\section{Discussion}

We have developed a strategy for quantifying time-resolved remodeling of protein-protein interactions in APs. The method provides quantitative data for confidently identifying true protein-protein interactions via comparisons with control APs, and notably, for following dynamic changes in protein-protein interactions in perturbed systems. We demonstrated the advantages in characterizing highly complex AP samples from a signaling related scaffold protein where the range of detected interacting protein abundances spans more than 4 orders of magnitude, and where $\sim 3 \%$ of the proteome is specifically co-purified. Since the 14-3-3 $\beta$ interactome represents one of the most complex interactomes known, centered around a single hub protein, we expect AP-SWATH to have general applicability in many quantitative protein interaction projects. Indeed, this is demonstrated in the accompanying article from Lambert et $a{ }^{46}$ who use AP-SWATH to quantify changes in protein-protein interactions associated with 
sequence variants or following drug treatment. While the applications differ significantly, from a methodological pespective our studies are similar, with both using a targeted extraction of DIA mass spectrometry to derive very complete quantitative interaction profiles in perturbed systems, albeit using a different set of software tools for data analysis and statistics.The dynamic interactome data allowed us to resolve distinct clusters, some of which are highly enriched in known or predicted substrates of AKT, and follow closely the profile of AKT activation. We also demonstrated the use of time-resolved quantitative AP-SWATH data in combination with known literature information to distinguish between the behavior of shared subunits from distinct protein complexes (mTORC1/2). This brings us closer to the goal of defining protein complexes present in the cell and how they are reorganized under perturbed conditions. These results differ substantially from the typical protein interaction network representations from static APMS studies. With that said, we would suggest that time-resolved protein abundance data from AP samples alone is unlikely to provide sufficient constraints to unambiguously define protein complexes in the cell and that analysis by large-scale reciprocal purification might not be feasible. Combination with orthogonal information, such as might be provided from improvements in native protein complex separation strategies ${ }^{47}$, or proximity labeling 48 , could prove instrumental toward this end.

AP-SWATH resembles AP-SRM 28 in terms of data consistency and quality, but with additional key advantages. Firstly, there is little overhead in terms of assay development as target assay libraries can be generated via side-by-side shotgun analysis and the once generated libraries can be perpetually reused. Secondly, there is no limit on the number of peptides that can be analyzed via AP-SWATH, whereas AP-SRM studies will struggle beyond 100 proteins. Finally, as APSWATH data contains an MS2 record for essentially the entire tryptic peptide space, there is potential for re-mining of the data post-acquisition using additional spectral libraries from sources such as synthetic peptides ${ }^{35,49}$, deep shotgun characterization, or enrichment of PTM containing peptides (Supplementary Results on phosphopeptide analysis). This type of iterative reanalysis is unique to SWATH or DIA data and provides a compelling argument for further development in this area.

The benefits of using targeted MS as a means to achieve high levels of completeness in quantitative proteomics are now widely appreciated ${ }^{50}$. SWATH-MS does not constitute a targeted MS method per se, but rather a targeted data analysis approach for DIA data which has been devised as a descendent of SRM with essentially the same goals in mind, albeit on an expanded scale. We have applied this strategy to the problem of describing dynamic reorganization of protein-protein interactions in perturbed systems. We believe that our APSWATH method could facilitate a shift towards more dynamic analyses in interaction proteomics 22 , with the eventual goal of answering questions relating to what degree of cellular environment sensing and functional control is mediated chiefly by reorganization of protein complexes and interaction networks.

\section{Access to data}

The mass spectrometry proteomics data have been deposited to the ProteomeXchange Consortium (http://proteomecentral.proteomexchange.org) via the PRIDE partner repository with the dataset identifier PXD000343. 


\section{Acknowledgements}

We thank P. Navarro for support with data analysis and spectral library generation; C. Ludwig, N. Selevsek, and Y. Liu for helpful discussions on SWATH-MS; O. Schubert for the MTB SWATHMS data file for target assay comparison; S. Hauri, A. van Drogen for advice on cell line generation, stimulation, and APs; A. Kahraman for discussions on structural aspects of 14-3-3; V. Chang for support with SRMstats; and the PRIDE team for assistance with upload of associated data. We gratefully acknowledge financial support from the SystemsX.ch project PhosphonetX and European Research Council advanced grant Proteomics v3.0 (grant 233226) from the European Union.

\section{Author Contributions}

B. C. performed the cell line generation, cell perturbation, AP and mass spectrometry experiments. L. G. co-developed the SWATH-MS methodology and provided critical input on analytical strategy. G. R. and H. R. developed the OpenSWATH software and provided critical input on SWATH-MS data analysis strategies. A. V. performed reciprocal AP-MS experiments. M. G. and R. A. conceived and co-supervised the project. B. C. wrote the manuscript with input from all authors.

\section{Competing financial interests}

The authors declare no competing financial interests

\section{References}

1. Hartwell, L.H., Hopfield, J.J., Leibler, S. \& Murray, A.W. From molecular to modular cell biology Nature 402, C47-52 (1999).

2. Robinson, C.V., Sali, A. \& Baumeister, W. The molecular sociology of the cell Nature 450, 973-982 (2007).

3. Pawson, T. Dynamic control of signaling by modular adaptor proteins Curr. Opin. Cell Biol. 19, 112-116 (2007).

4. Good, M.C., Zalatan, J.G. \& Lim, W.A. Scaffold proteins: hubs for controlling the flow of cellular information Science 332, 680-686 (2011).

5. Chen, S., Synowsky, S., Tinti, M. \& MacKintosh, C. The capture of phosphoproteins by 143-3 proteins mediates actions of insulin Trends Endocrinol. Metab. 22, 429-436 (2011).

6. Jin, J. et al. Proteomic, functional, and domain-based analysis of in vivo 14-3-3 binding proteins involved in cytoskeletal regulation and cellular organization Curr. Biol. 14, 1436-1450 (2004).

7. Ballif, B.A. et al. Identification of 14-3-3epsilon substrates from embryonic murine brain J. Proteome Res. 5, 2372-2379 (2006).

8. He, Y.F. et al. Biotin tagging coupled with amino acid-coded mass tagging for efficient and precise screening of interaction proteome in mammalian cells Proteomics 9, 5414-5424 (2009).

9. Johnson, C. et al. Visualization and biochemical analyses of the emerging Mammalian 143-3-phosphoproteome Mol. Cell. Proteomics 10, M110 005751 (2011). 
10. Rinner, 0. et al. An integrated mass spectrometric and computational framework for the analysis of protein interaction networks Nat. Biotechnol. 25, 345-352 (2007).

11. Dubois, F. et al. Differential 14-3-3 affinity capture reveals new downstream targets of phosphatidylinositol 3-kinase signaling Mol. Cell. Proteomics 8, 2487-2499 (2009).

12. Larance, M. et al. Global phosphoproteomics identifies a major role for AKT and 14-3-3 in regulating EDC3 Mol. Cell. Proteomics 9, 682-694 (2010).

13. Gingras, A.C., Gstaiger, M., Raught, B. \& Aebersold, R. Analysis of protein complexes using mass spectrometry Nat. Rev. Mol. Cell Biol. 8, 645-654 (2007).

14. Gavin, A.C., Maeda, K. \& Kuhner, S. Recent advances in charting protein-protein interaction: mass spectrometry-based approaches Curr. Opin. Biotechnol. 22, 42-49 (2010).

15. Gavin, A.C. et al. Proteome survey reveals modularity of the yeast cell machinery Nature 440, 631-636 (2006).

16. Krogan, N.J. et al. Global landscape of protein complexes in the yeast Saccharomyces cerevisiae Nature 440, 637-643 (2006).

17. Kuhner, S. et al. Proteome organization in a genome-reduced bacterium Science 326, 1235-1240 (2009).

18. Glatter, T., Wepf, A., Aebersold, R. \& Gstaiger, M. An integrated workflow for charting the human interaction proteome: insights into the PP2A system Mol Syst Biol 5, 237 (2009).

19. Hubner, N.C. et al. Quantitative proteomics combined with BAC TransgeneOmics reveals in vivo protein interactions J. Cell Biol. 189, $739-754$ (2010).

20. Sowa, M.E., Bennett, E.J., Gygi, S.P. \& Harper, J.W. Defining the human deubiquitinating enzyme interaction landscape Cell 138, 389-403 (2009).

21. Varjosalo, M. et al. Interlaboratory reproducibility of large-scale human protein-complex analysis by standardized AP-MS Nat Methods 10, 307-314 (2013).

22. Ideker, T. \& Krogan, N.J. Differential network biology Mol Syst Biol 8, 565 (2012).

23. Wepf, A. et al. Quantitative interaction proteomics using mass spectrometry Nat Methods 6, 203-205 (2009).

24. von Kriegsheim, A. et al. Cell fate decisions are specified by the dynamic ERK interactome Nat. Cell Biol. 11, 1458-1464 (2009).

25. Bennett, E.J., Rush, J., Gygi, S.P. \& Harper, J.W. Dynamics of cullin-RING ubiquitin ligase network revealed by systematic quantitative proteomics Cell 143, 951-965 (2010).

26. Glatter, T. et al. Modularity and hormone sensitivity of the Drosophila melanogaster insulin receptor/target of rapamycin interaction proteome Mol Syst Biol 7, 547 (2011).

27. Domon, B. \& Aebersold, R. Options and considerations when selecting a quantitative proteomics strategy Nat. Biotechnol. 28, 710-721 (2010).

28. Bisson, N. et al. Selected reaction monitoring mass spectrometry reveals the dynamics of signaling through the GRB2 adaptor Nat. Biotechnol. 29, 653-658 (2011).

29. Zheng, Y. et al. Temporal regulation of EGF signalling networks by the scaffold protein Shc1 Nature 499, 166-171 (2013).

30. Purvine, S., Eppel, J.T., Yi, E.C. \& Goodlett, D.R. Shotgun collision-induced dissociation of peptides using a time of flight mass analyzer Proteomics 3, 847-850 (2003).

31. Venable, J.D. et al. Automated approach for quantitative analysis of complex peptide mixtures from tandem mass spectra Nat Methods 1, 39-45 (2004).

32. Plumb, R.S. et al. UPLC/MS(E); a new approach for generating molecular fragment information for biomarker structure elucidation Rapid Commun. Mass Spectrom. 20, 1989-1994 (2006).

33. Panchaud, A. et al. Precursor acquisition independent from ion count: how to dive deeper into the proteomics ocean Anal. Chem. 81, 6481-6488 (2009).

34. Egertson, J.D. et al. Multiplexed MS/MS for improved data-independent acquisition Nat Methods 10, 744-746 (2013).

35. Gillet, L.C. et al. Targeted data extraction of the MS/MS spectra generated by data independent acquisition: a new concept for consistent and accurate proteome analysis Mol. Cell. Proteomics 11, 0111.016717 (2012).

36. Reiter, L. et al. mProphet: automated data processing and statistical validation for largescale SRM experiments Nat Methods 8, 430-435 (2011). 
37. Lam, H. et al. Development and validation of a spectral library searching method for peptide identification from MS/MS Proteomics 7, 655-667 (2007).

38. Chang, C.Y. et al. Protein significance analysis in selected reaction monitoring (SRM) measurements Mol. Cell. Proteomics 11, M111 014662 (2012).

39. Kovacina, K.S. et al. Identification of a proline-rich Akt substrate as a 14-3-3 binding partner J. Biol. Chem. 278, 10189-10194 (2003).

40. Linding, R. et al. Systematic discovery of in vivo phosphorylation networks Cell 129, 1415-1426 (2007).

41. Laplante, M. \& Sabatini, D.M. mTOR signaling at a glance J. Cell Sci. 122, 3589-3594 (2009).

42. Dibble, C.C., Asara, J.M. \& Manning, B.D. Characterization of Rictor phosphorylation sites reveals direct regulation of mTOR complex 2 by S6K1 Mol. Cell. Biol. 29, 5657-5670 (2009).

43. Silva, J.C. et al. Absolute quantification of proteins by LCMSE: a virtue of parallel MS acquisition Mol. Cell. Proteomics 5, 144-156 (2006).

44. Liu, Y. et al. Quantitative Measurements of N-linked Glycoproteins in Human Plasma by SWATH-MS Proteomics 13, 1247-1256 (2013).

45. Ludwig, C., Claassen, M., Schmidt, A. \& Aebersold, R. Estimation of absolute protein quantities of unlabeled samples by selected reaction monitoring mass spectrometry Mol. Cell. Proteomics 11, M111 013987 (2012).

46. Lambert, J. P., et al. Mapping differential interactomes by affinity purification coupled with data independent mass spectrometry acquisition Nat Methods, in press (2013).

47. Kristensen, A.R., Gsponer, J. \& Foster, L.J. A high-throughput approach for measuring temporal changes in the interactome Nat Methods 9, 907-909 (2012).

48. Roux, K.J., Kim, D.I., Raida, M. \& Burke, B. A promiscuous biotin ligase fusion protein identifies proximal and interacting proteins in mammalian cells J. Cell Biol. 196, 801-810 (2012). 49. Picotti, P. et al. A complete mass-spectrometric map of the yeast proteome applied to quantitative trait analysis Nature 494, 266-270 (2013).

50. Marx, V. Targeted proteomics Nat. Meth. 10, 19-22 (2013).

\section{Figure Legends}

\section{Figure 1 - AP-SWATH workflow schematic}

(a) 14-3-3 $\beta$ is affinity purified under native conditions over a time course which includes the following conditions: no treatment, $\mathrm{t}=-60 \mathrm{~min}$; after PI3K inhibitor pre-treatment, $\mathrm{t}=0 \mathrm{~min}$; and 4 time points after IGF1 stimulation, $\mathrm{t}=1 \mathrm{~min}, \mathrm{t}=10 \mathrm{~min} ; \mathrm{t}=30 \mathrm{~min}$, and $\mathrm{t}=100 \mathrm{~min}$. (b) Samples analyzed by AP are analyzed in parallel by SWATH-MS and shotgun MS. The shotgun data is searched against a protein database using standard methods and a peptide spectral library is constructed. Target assays containing the most abundant fragment ions from the spectral libraries are used to extract MS2 level ion chromatograms from the SWATH-MS data. The resulting peak groups are scored using a decoy model. Quantitative values are then used to confirm specific 14-3-3 protein interactions. Finally, the protein abundance values over the time course perturbation are used to interpret protein interaction dynamics.

\section{Figure 2 - Complete quantitative data matrix from AP-SWATH}

Quantitative data for 1,967 proteins was extracted from AP-SWATH data for 27 samples including $18 \times 14-3-3 \beta$ AP samples and $9 \times$ GFP control AP samples. The proteins were clustered in the vertical direction hierarchically using Minkowski distance. This data matrix formed the 
basis for all downstream quantitative comparisons including filtering of non-specific contaminant proteins (Fig. 3) and time course protein interaction dynamics (Fig. 4).

Figure 3 - 14-3-3 $\beta$ protein interactions are confidently identified by enrichment over control APs

(a) A volcano plot showing $\log 2$ fold change is plotted against - $\log 10$ adjusted p-value for 14-3$3 \beta$ AP samples versus samples generated from an irrelevant bait (GFP). Data points highlighted in red in the upper right section represent proteins which display and enrichment in 14-3-3 $\beta$ APs of $\log 2$ fold change $>2$ and adjusted p-value $<0.01$. (b) A subset of the detected protein interactions were verified by reciprocal AP-MS. 14-3-3 $\beta$ (red circles) was detected as a protein interaction in 19 of the 21 protein kinases analysed. The bait abundances (black circles) of the negative control kinase group spanned the same abundance range as the set of detected 14-3-3 $\beta$ interactors. The number of replicates is indicated in brackets and the error bars represent $+/-1$ standard deviation.

\section{Figure 4 - Quantification of 14-3-3 $\beta$ interactome after perturbation}

Panels (a) - (f) show the time course behavior of 6 representative 14-3-3 interacting proteins. Panels (g) - (h) show the 5 distinct time course clusters found using fuzzy c-means clustering of the time series data. The 5 selected proteins in panels (a) - (e) are typical of the 5 clusters found in panels (g) - (h). IGF1R shown in panel (f) is nominally part of cluster 2, however, the time course behavior is not completely consistent as IGF1R shows no response to the PI3K inhibitor unlike the other proteins shown in cluster 2. Panel (i) shows the enrichment of known or predicted AKT substrates in each of the 5 clusters compared to the background UniProt human proteome.

\section{Figure 5 - AP-SWATH time-resolved data highlights sequestering of the mTORC1 inhibitory subunit AKTS1 but no change in mTORC2 bound to 14-3-3 $\beta$}

(a) sub-network of 14-3-3 $\beta$ interacting proteins overlaid on PINA literature interactions centered on mTOR kinase and nearest neighbors. (b) Abundances of proteins from (a) across the time course. (c) Representative AP-SWATH data for the AKTS1 peptide containing the phosphorylated threonine 246 in the known 14-3-3 binding motif. (d) Time course abundance of

the same AKTS1 phosphopeptide from (c) which matches exactly the dynamic behavior of AKTS1 in (b). (e) Schematic summary of the 14-3-3 $\beta$ dynamic interaction data combined with previously known literature information.

\section{Figure 6 - Dynamic range of 14-3-3 $\beta$ interacting proteins}

The $\log 10$ abundance of 567 high confidence 14-3-3 $\beta$ interacting proteins calculated using the 'best flyer peptide' approach is plotted (black circles). The same set of proteins is re-plotted with an offset 4 additional times to highlight 14-3-3 protein isoforms (purple circles), proteins containing a 14-3-3 ligand motif (blue circles), 14-3-3 $\beta$ protein interactions previously detected 
in the literature (turquoise circles), and the set of protein kinases verified by reciprocal AP-MS (orange circles).

\section{Online Methods}

\section{Cell line generation}

HEK293 cells (Invitrogen) inducibly expressing n-terminally SH tagged (tandem streptavidin binding peptide tag + hemagglutinin tag) $14-3-3 \beta$ were generated essentially as described previously51. (Cells have not been recently tested for mycoplasma contamination). The 14-3-3 $\beta$ ORF, obtained in pDONR223 from the Gateway compatible human orfeome library (hORFeome v5.1, Open Biosystems), was recombined into the pcDNA5/FRT/TO/SH/GW destination vector using the LR Clonase II kit (Life Technologies). Flp-In T-REx HEK293 cells (Life Technologies) containing a single genomic FRT site and stably expressing the tet repressor were cultured in DMEM (10 \% FCS, $50 \mu \mathrm{g} / \mathrm{ml}$ penicillin, $50 \mu \mathrm{g} / \mathrm{ml}$ streptomycin), and co-transfected using Fugene 6 transfection reagent (Promega) with the 14-3-3 $\beta$ expression plasmid and p0G44 vector (Life Technologies) for coexpression of the Flp-recombinase. After 2 days cells were selected using media containing $130 \mu \mathrm{g} / \mathrm{ml}$ hygromycin and $19 \mu \mathrm{g} / \mathrm{ml}$ blasticidin for 2-3 weeks and expanded. Bait expression was checked by western blotting against the HA tag (HA.11 monoclonal antibody, Covance, dilution 1:3,000) and 14-3-3 $\beta$ (Atlas antibodies, dilution 1:500).

\section{Time course treatment of cells}

Expression of the bait in $40 \%$ confluent cells was induced for 24 hours with media containing $1.33 \mu \mathrm{g} / \mathrm{ml}$ doxycycline (Sigma). The cells were then washed in PBS, and then serum starved overnight with serum-free DMEM containing doxycycline. The time course was started $(\mathrm{t}=-60$ min) when sub-confluent cells were pre-treated with the reversible phosphatidylinositol 3kinase inhibitor LY294002 (Life Technologies) at $20 \mu \mathrm{M}$ (final concentration) for one hour [Note: LY294002 was included in the time course experiment because serum starvation of Flp-In T-REx HEK293 cells was not sufficient to significantly reduce AKT activity, as judged by p-AKT S473 Western blotting, Cell Signaling, antibody dilution 1:3,000]. After this pre-treatment, the cells were washed briefly with PBS ( $\mathrm{t}=0 \mathrm{~min}$ ) and treated with $50 \mathrm{ng} / \mathrm{ml}$ IGF1 (Sigma) in serum free DMEM with doxycycline for 1, 10, 30, or 100 minutes. In total, cells from 6 time points were collected (see Fig 1). Cells were immediately harvested on ice by pipetting in ice-cold PBS with 1 mM EDTA, pelleted by centrifugation for 3 minutes at $300 \mathrm{xg}$ at $4{ }^{\circ} \mathrm{C}$, and snap frozen. The time course treatment was carried out in biological triplicate, using $2 \times 15 \mathrm{~cm}$ dishes per condition, in serial passages of the HEK-Flp-1433B cells.

\section{Affinity purification}

The 14-3-3 $\beta$ protein complexes were obtained by single step AP via the tandem streptavidin binding peptide sequence included in the SH tag. Cells were lysed on ice in HNN lysis buffer (0.5\% NP40, 50mM HEPES, pH 7.5, 150mM NaCl, 50mM NaF, $200 \mu \mathrm{M} \mathrm{NaV03,} \mathrm{0.5mM} \mathrm{PMSF,} 1.2$ 
$\mu \mathrm{M}$ Avidin, and protease inhibitor cocktail (Sigma)) and centrifuged at 16,100 x g for 15 minutes at $4{ }^{\circ} \mathrm{C}$. The cleared lysate was incubated with $50 \mu \mathrm{l}$ of Strep-Tactin Sepharose (Iba) at $4{ }^{\circ} \mathrm{C}$ with mixing for 15 minutes. The lysate/bead mixture was loaded onto an empty spin column (BioRad) and the beads were washed with $2 \times 1 \mathrm{ml}$ HNN lysis buffer, and $3 \times 1 \mathrm{ml}$ HNN buffer (50mM HEPES, pH 7.5, $150 \mathrm{mM} \mathrm{NaCl}, 50 \mathrm{mM} \mathrm{NaF}$ ) by gravity flow. Bait complexes were released from the affinity matrix with $3 \times 200 \mu \mathrm{lNN}$ elution buffer $(2.5 \mathrm{mM}$ biotin, 50mM HEPES, pH 7.5, $150 \mathrm{mM} \mathrm{NaCl}, 50 \mathrm{mM} \mathrm{NaF}$ ). Protein from the eluate was precipitated by addition of TCA to $25 \%$ (v/v) and incubation on ice for 1 hour. Protein was pelleted by centrifugation at 16,100 x g for 15 minutes at $4{ }^{\circ} \mathrm{C}$. Protein pellets were washed 3 times with $200 \mu \mathrm{l}$ ice cold acetone with interspersed centrifugation, dried briefly by vacuum centrifugation at $45^{\circ} \mathrm{C}$, and resuspended in $8 \mathrm{M}$ urea, $50 \mathrm{mM} \mathrm{NH}_{4} \mathrm{HCO}_{3}$. Cysteines were reduced (5 mM TCEP, 30 minutes) and alkylated (10 $\mathrm{mM}$ iodoacetamide, 30 minutes), and the urea concentration was reduced to $1.5 \mathrm{M}$ by dilution

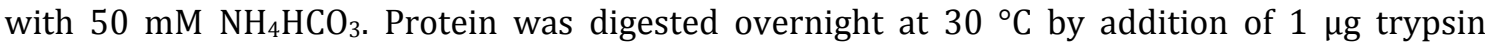
(Promega, sequencing grade). Formic acid was added to $1.25 \%(\mathrm{v} / \mathrm{v})$ and peptides were purified using C18 microspin columns (Nest Group) and resuspended in $12 \mu \mathrm{l}$ buffer A containing iRT peptides for retention time alignment (Biognosys) and an AQUA heavy peptide standard (Thermo) corresponding to a tryptic peptide from the affinity tag52 (AADITSLYK* $-250 \mathrm{fmol} / \mu \mathrm{l}$ ), and $4 \mu \mathrm{l}$ were injected serially in SWATH and shotgun acquisition modes. APs for phosphoenrichment were as above, except that forty culture plates of $15 \mathrm{~cm}$ diameter were used as starting material. Phosphopeptides were enriched from tryptic digests on IMAC-Fe resin as described ${ }^{53}$. Reciprocal APs of protein kinases were as described above, except that $8 \times 15 \mathrm{~cm}$ dishes were used per purification and cells were grown under serum fed conditions (10\% FBS). Dynamics for 6 selected 14-3-3 $\beta$ interactors was verified by Western blotting (antibody dilutions: AKTS1 - 1:1000, TBCD4 - 1:100, CISY - 1:250, FBSP1 - 1:200, F262 - 1:100, RAE1L 1:100, HA - 1:5,000, see Supplementary Figure 7 for further antibody details).

\section{SWATH mass spectrometry}

SWATH mass spectra were acquired using an AB Sciex 5600 TripleTOF mass spectrometer interfaced to an Eksigent NanoLC Ultra 2D Plus HPLC system essentially as previously described ${ }^{54}$. Samples were chromatographed using a 120 minute gradient from 2-35 \% (buffer A $0.1 \%(\mathrm{v} / \mathrm{v})$ formic acid, $2 \%(\mathrm{v} / \mathrm{v})$ acetonitrile, buffer B $0.1 \%(\mathrm{v} / \mathrm{v})$ formic acid, $90 \%(\mathrm{v} / \mathrm{v})$ acetonitrile) after direct injection on to a $20 \mathrm{~cm}$ PicoFrit emitter (New Objective) packed to 20 cm with Magic C18 AQ $3 \mu \mathrm{m} 200 \AA$ stationary phase. For SWATH-MS-based experiments, the mass spectrometer was operated in a looped product ion mode. The instrument was specifically tuned to allow a quadrupole resolution of $25 \mathrm{~m} / \mathrm{z}$ mass selection. Using an isolation width of 26 $\mathrm{m} / \mathrm{z}$ (containing $1 \mathrm{~m} / \mathrm{z}$ for the window overlap), a set of 32 overlapping windows was constructed covering the precursor mass range of 400-1200 m/z. The effective isolation windows can be considered as being 399.5-424.5, 425.5-449.5, etc. (plus the overlaps from the nominal window transmission). SWATH MS2 spectra were collected from 100-2000 m/z. The collision energy for each window was determined based on the calculation for a charge $2+$ ion centered upon the window with a spread of 15. An accumulation time of $100 \mathrm{~ms}$ was used for all fragment ion scans in high sensitivity mode and for the survey scans in high resolution mode acquired at the beginning of each cycle, resulting in a duty cycle of $\sim 3.4 \mathrm{~s}$. 
In addition to SWATH-MS acquisition, every sample was also analysed using classical shotgun data acquisition with a TripleTOF 5600. The chromatographic parameters were as above for SWATH-MS. MS1 spectra were collected in the range 360-1460 m/z for $250 \mathrm{~ms}$. The 20 most intense precursors with charge state 2-5 were selected for fragmentation and MS2 spectra were collected in the range $50-2000 \mathrm{~m} / \mathrm{z}$ for $100 \mathrm{~ms}$, and precursor ions were excluded from reselection for 15 seconds. Reciprocal APs of protein kinases were analysed using an Orbitrap Elite with MS1 resolving power set to 240,000 and the top 10 precursors selected for MS2 in the ion trap after CID activation. Peptides were separated on an Acclaim PepMap100 $2 \mu \mathrm{m}$ C18 15 $\mathrm{cm} \times 75 \mu \mathrm{m}$ using a 45 minute gradient at $300 \mathrm{nl} / \mathrm{min}$ from a EASY-nLC 1000 (Thermo Scientific).

\section{Spectral library and target assay construction}

Profile mode wiff files from shotgun data acquisition were centroided and converted to mzML using the AB Sciex Data Converter v1.3, and further converted to mzXML using ProteoWizard55 MSConvert v3.04.238. Mass spectra were queried against the canonical UniProt complete proteome database for human (July 2011) appended with common contaminants and reversed sequence decoys (40,548 protein sequences including decoys) using XTandem with kscore plugin $^{56}$, and additionally XTandem with native scoring57. Carbamidomethyl was set as fixed modification for cysteines, and methionine oxidation and phosphorylation on serine/threonine/tyrosine were set as variable modification. Semi-tryptic peptides and peptides with up to 2 missed cleavages were allowed, and mass error was set as $30 \mathrm{ppm}$ and $75 \mathrm{ppm}$ for precursor and product ions respectively. Results from both search engines were individually converted to the pepXML format using Tandem2XML v4.6.0, analysed with PeptideProphet ${ }^{58}$ v4.6.0, and combined using iProphet ${ }^{59}$ v4.6.0 including false identification rate calculation. Phosphosite localisation probabilities were estimated using PTMProphet v4.6.1. Reciprocal purifications analysed on the Orbitrap Elite were searched using XTandem with kscore as above except mass accuracies were 25 ppm and 0.5 Da for MS1 and MS2 respectively.

A redundant spectral library (i.e. still retaining all valid peptide spectrum matches) was generated from the iProphet results using SpectraST60 v4.6.0. Retention times for peptide identifications in the redundant spectral library were mapped into the iRT ${ }^{61}$ space with reference to a linear calibration constructed for each shotgun run using the spiked iRT peptides, and the median iRT was calculated for each peptide in the spectral library using a custom python script (spectrast2spectrast_irt.py) [Note: All Python scripts used are available at https://code.google.com/p/msproteomicstools/]. A non-redundant consensus spectral library was then constructed from the redundant library using SpectraST.

To obtain assays for SWATH-MS targeted extraction, the 5 most abundant b or y fragment ions for each peptide precursor were selected from the non-redundant consensus spectral library in the range $400-2000 \mathrm{~m} / \mathrm{z}$ and excluding the precursor isolation window using a custom python script (spectrast2peakview.py). The assay list was outputted to a .tsv file and converted to TraML format, a standard XML based format for transition list exchange, using the OpenSWATH tool ConvertTSVToTraML. Sequence shuffled decoys were appended to the assays using the OpenSWATH tool OpenSwathDecoyGenerator. 


\section{SWATH-MS targeted data extraction}

SWATH-MS wiff files were first converted to profile mzXML using Proteowizard msconvert 55 v3.0.3316. As the precursor isolation window scheme was wrongly retrived by msconvert, a custom python script (fix_swath_windows.py) was used to correct this. For easier file access, the mzXML files were split into 33 individual files (32 SWATH MS2 files +1 MS1 file) using the custom python script (split_mzXML_intoSwath.py). These files were then converted to profile mzML and gzipped for use in further analysis.

The SWATH targeted data analysis was carried out using OpenSWATH r10367 (Röst et al; submitted manuscript; for a tutorial and test data set see www.openswath.org) running on an internal computing cluster and consisted of the following steps. Fragment ion chromatograms were extracted for each peptide precursor in its appropriate SWATH-MS data file based on the

TraML format target and decoy assays with a width of $0.02 \mathrm{~m} / \mathrm{z}$ (OpenSwathChromatogramExtractor). Additionally, ion chromatograms for the iRT retention time standard peptides were extracted to facilitate linear retention alignment (OpenSwathRTNormalizer). Peak groups from the extracted fragment ion chromatograms were formed and scored based on their elution profiles, similarity to the target assay in terms of RT and relative fragment ion intensity, as well as features from the full MS2 SWATH spectrum extracted at the chromatographic peak apex (OpenSwathAnalyzer). Finally, the optimal separation between true and false peak groups was achieved using a linear model training and the score distribution from the shuffled decoy assays was used to estimate the false discovery rate (mProphet ${ }^{62}$ ).

Identification of phosphopeptides from AP-SWATH data using phosphopeptide specific libraries was also performed using OpenSWATH. However, in this case we did not perform automated quantification for all detected phosphopeptides due to the potential for errors from mismatching isobaric phosphopeptides which frequently share a majority of fragment ions. Tools to resolve such cases and determine more accurately phosphosite localization in SWATH data are under development (Gillet L., Maiolica A., et al., 2013, Proceedings of the 61st ASMS Conference on Mass Spectrometry, Minneapolis). As such, extraction and manual inspection of selected phosphopeptide signals (as in Figure 5d) from AP-SWATH data was performed by using Skyline ${ }^{3}$ v1.4.1.4646 (see also Supplementary Results on phosphopeptide analysis).

\section{Data analysis and statistics}

Peptide features (i.e. peptides in a given charge state) which met the $1 \%$ FDR threshold in 3 out of 3 biological replicates for any experimental condition were retained, and intensities for these peptides/transitions across the experiment were used for further analysis. 14-3-3 $\beta$ APs in biological triplicate were compared to corresponding triplicate GFP purifications to confidently identify true protein interactions. Protein level statistical significance and fold changes between 14-3-3 $\beta$ purifications and GFP control purifications, as well as between 14-3-3 purifications at time points in the perturbation experiment, were calculated using a linear mixed effects model implemented in the open source R package SRMstats ${ }^{64}$ (see Supplementary Note 2), which uses transition level intensities to model protein level abundance changes. The 2 most intense transitions (in any charge state) from the 3 most intense peptides for a protein were used as 
input for protein level inference calculations in SRMstats. For comparisons between 14-3-3 $\beta$ and GFP control purifications, data were not normalized. For comparisons between 14-3-3 $\beta$ purifications across the time course, abundances were normalized with respect the 3 most abundant tryptic peptides from the affinity tag2. For statistical analyses in the time course, all comparisons were made to the $2^{\text {nd }}$ time point ( $\mathrm{t}=0$ minutes). Protein abundances were estimated by summing the 2 most intense transitions from the 3 most intense peptides for a protein as previously described for SRM data65. Gene ontology and domain enrichment were performed using the DAVID Bioinformatics Resources ${ }^{66}$. We used a non-strict filter on the quantitative data to remove proteins which showed no change $(-0.5>\log 2 \mathrm{FC}>0.5$, adjusted pvalue $<0.05$ at any time point compared with $t=0$ minutes), and then performed time series clustering using the open source R package $\mathrm{Mfuzz}^{67}$ using median fold change as input. The list of AKT substrates used for enrichment analysis was constructed by combining a literature curated list of AKT substrates obtained from PhosphoSitePlus 68 with predicted substrates from NetworKIN69. Protein abundance estimates using the 'best flyer peptide' approach were calculated by summing the 2 most intense transitions for the 3 most intense peptides as previously described for SRM data ${ }^{65}$. Literature protein-protein interactions were retrieved from the PINA ${ }^{70}$ and iRefWeb ${ }^{71}$ interaction databases.

51. Glatter, T., Wepf, A., Aebersold, R. \& Gstaiger, M. An integrated workflow for charting the human interaction proteome: insights into the PP2A system Mol Syst Biol 5, 237 (2009).

52. Wepf, A. et al. Quantitative interaction proteomics using mass spectrometry Nat Methods 6, 203-205 (2009).

53. Villen, J. \& Gygi, S.P. The SCX/IMAC enrichment approach for global phosphorylation analysis by mass spectrometry Nature protocols 3, 1630-1638 (2008).

54. Gillet, L.C. et al. Targeted data extraction of the MS/MS spectra generated by data independent acquisition: a new concept for consistent and accurate proteome analysis Mol. Cell. Proteomics (2012).

55. Kessner, D. et al. ProteoWizard: open source software for rapid proteomics tools development Bioinformatics 24, 2534-2536 (2008).

56. MacLean, B., Eng, J.K., Beavis, R.C. \& McIntosh, M. General framework for developing and evaluating database scoring algorithms using the TANDEM search engine Bioinformatics 22, 2830-2832 (2006).

57. Craig, R. \& Beavis, R.C. TANDEM: matching proteins with tandem mass spectra Bioinformatics 20, 1466-1467 (2004).

58. Keller, A., Nesvizhskii, A.I., Kolker, E. \& Aebersold, R. Empirical statistical model to estimate the accuracy of peptide identifications made by MS/MS and database search Anal. Chem. 74, 5383-5392 (2002).

59. Shteynberg, D. et al. iProphet: multi-level integrative analysis of shotgun proteomic data improves peptide and protein identification rates and error estimates Mol. Cell. Proteomics 10, M111 007690 (2011).

60. Lam, H. et al. Development and validation of a spectral library searching method for peptide identification from MS/MS Proteomics 7, 655-667 (2007).

61. Escher, C. et al. Using iRT, a normalized retention time for more targeted measurement of peptides Proteomics 12,1111-1121 (2012).

62. Reiter, L. et al. mProphet: automated data processing and statistical validation for largescale SRM experiments Nat Methods (2011).

63. Maclean, B. et al. Skyline: An Open Source Document Editor for Creating and Analyzing Targeted Proteomics Experiments Bioinformatics 26 (2010). 
64. Chang, C.Y. et al. Protein significance analysis in selected reaction monitoring (SRM) measurements Mol. Cell. Proteomics 11, M111 014662 (2012).

65. Ludwig, C., Claassen, M., Schmidt, A. \& Aebersold, R. Estimation of absolute protein quantities of unlabeled samples by selected reaction monitoring mass spectrometry Mol. Cell. Proteomics 11, M111 013987 (2012).

66. Huang da, W., Sherman, B.T. \& Lempicki, R.A. Systematic and integrative analysis of large gene lists using DAVID bioinformatics resources Nature protocols 4, 44-57 (2009).

67. Futschik, M.E. \& Carlisle, B. Noise-robust soft clustering of gene expression time-course data J. Bioinform. Comput. Biol. 3, 965-988 (2005).

68. Hornbeck, P.V. et al. PhosphoSite: A bioinformatics resource dedicated to physiological protein phosphorylation Proteomics 4, 1551-1561 (2004).

69. Linding, R. et al. Systematic discovery of in vivo phosphorylation networks Cell 129, 1415-1426 (2007).

70. $\mathrm{Wu}$, J. et al. Integrated network analysis platform for protein-protein interactions Nat Methods 6, 75-77 (2009).

71. Turner, B. et al. iRefWeb: interactive analysis of consolidated protein interaction data and their supporting evidence Database : the journal of biological databases and curation 2010, baq023 (2010). 
a

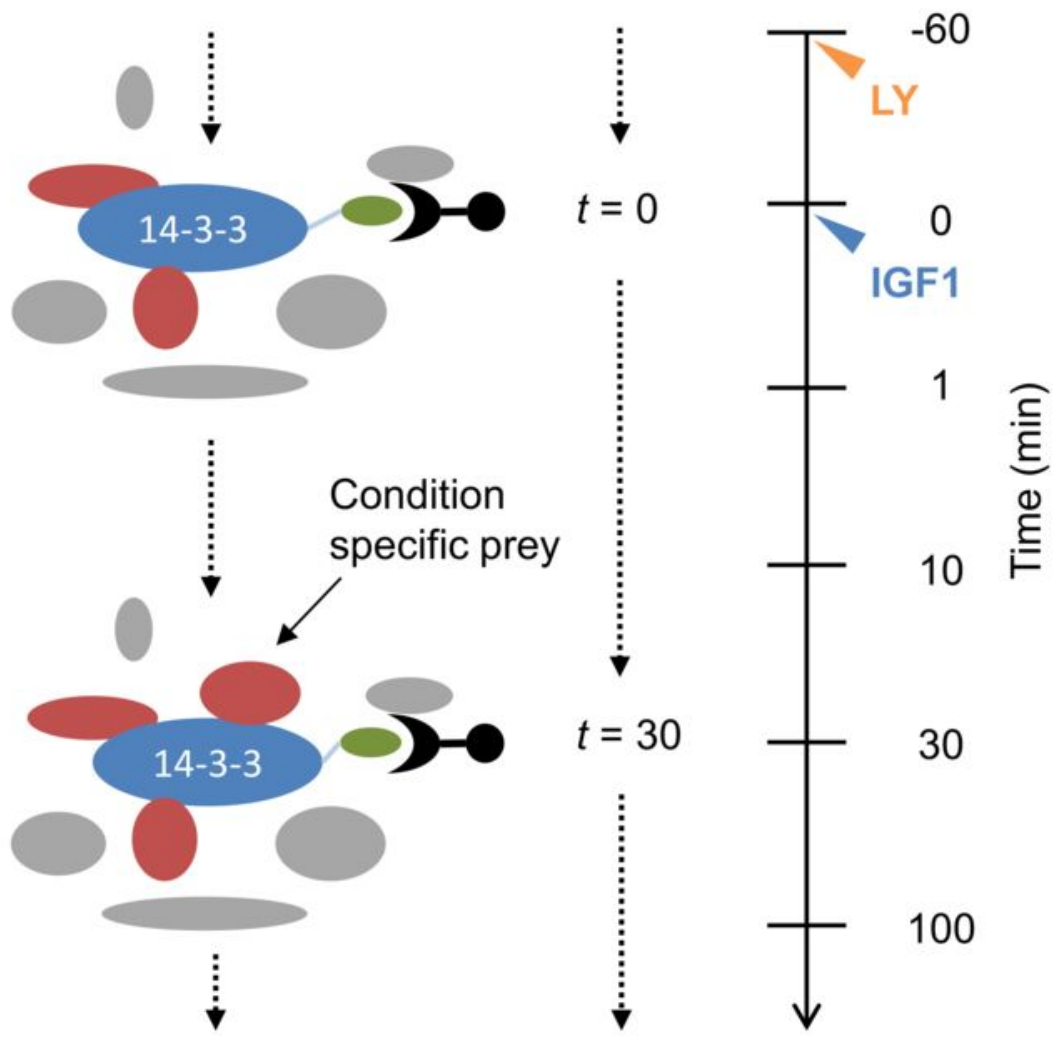


b

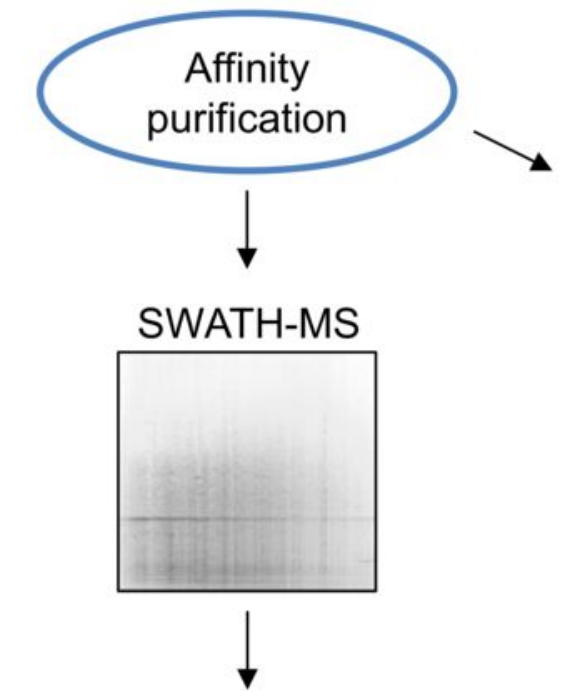

\section{Shotgun-MS}

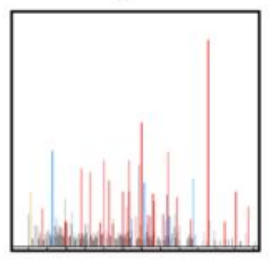

Targeted extraction

Error model

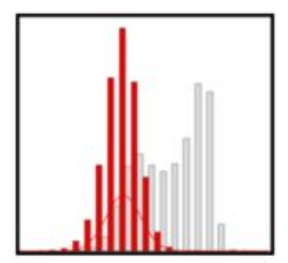

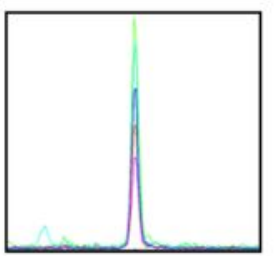

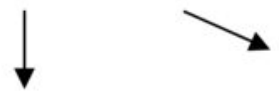

High confidence protein interactions

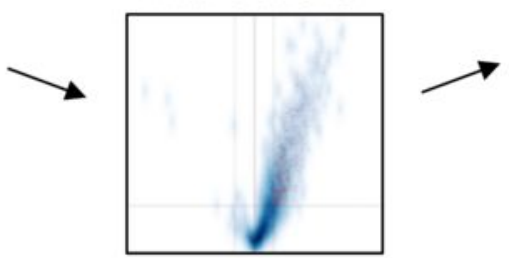

Spectral library/ target assays

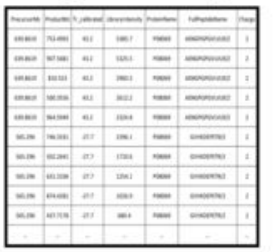

\section{Protein} interaction dynamics 
Time (min)

$14-3-3 \beta$

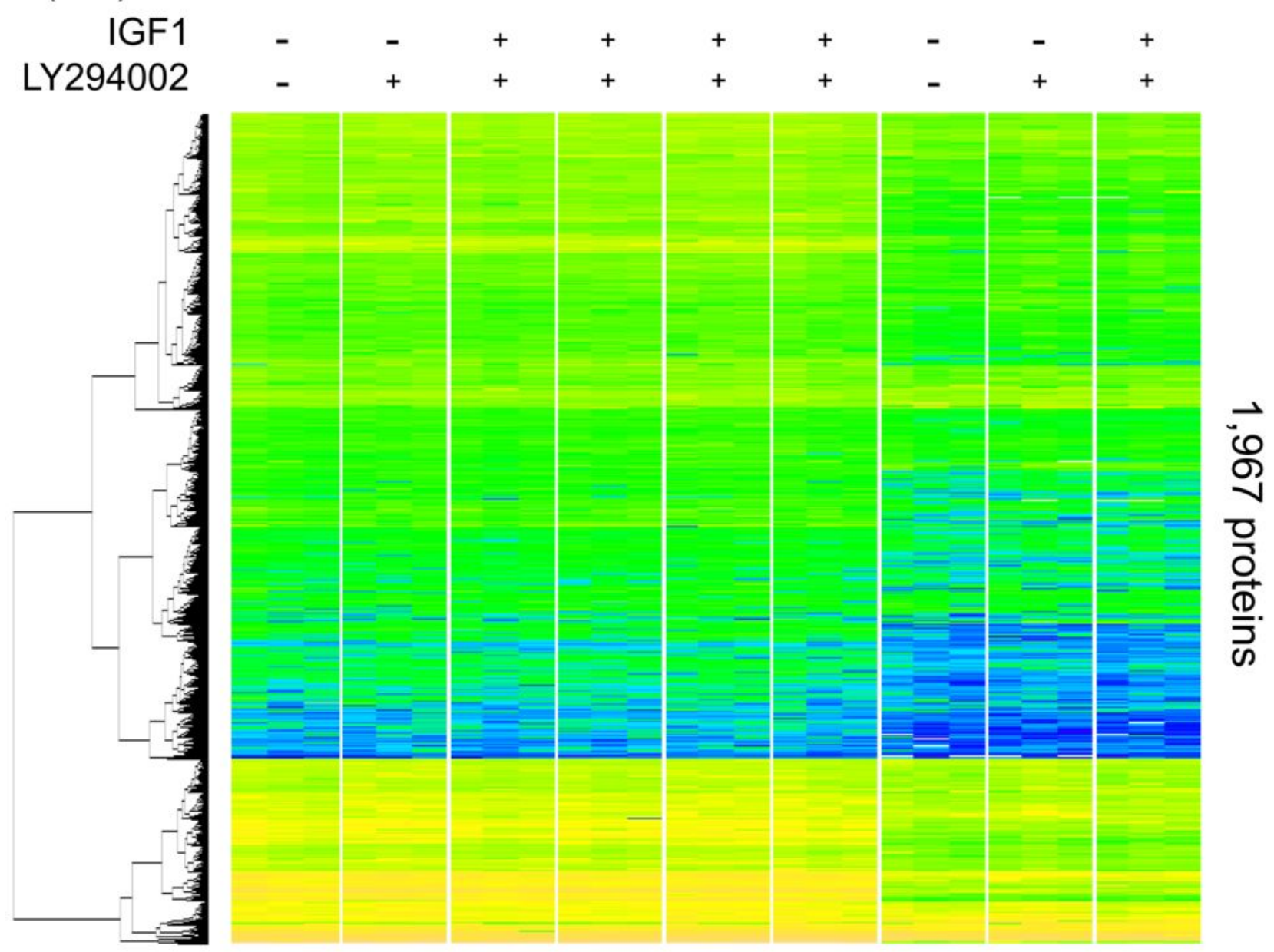

$\begin{array}{llll}5 & 10 & 15 \quad 20\end{array}$

$\log _{2}$ protein abundance

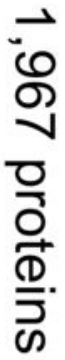




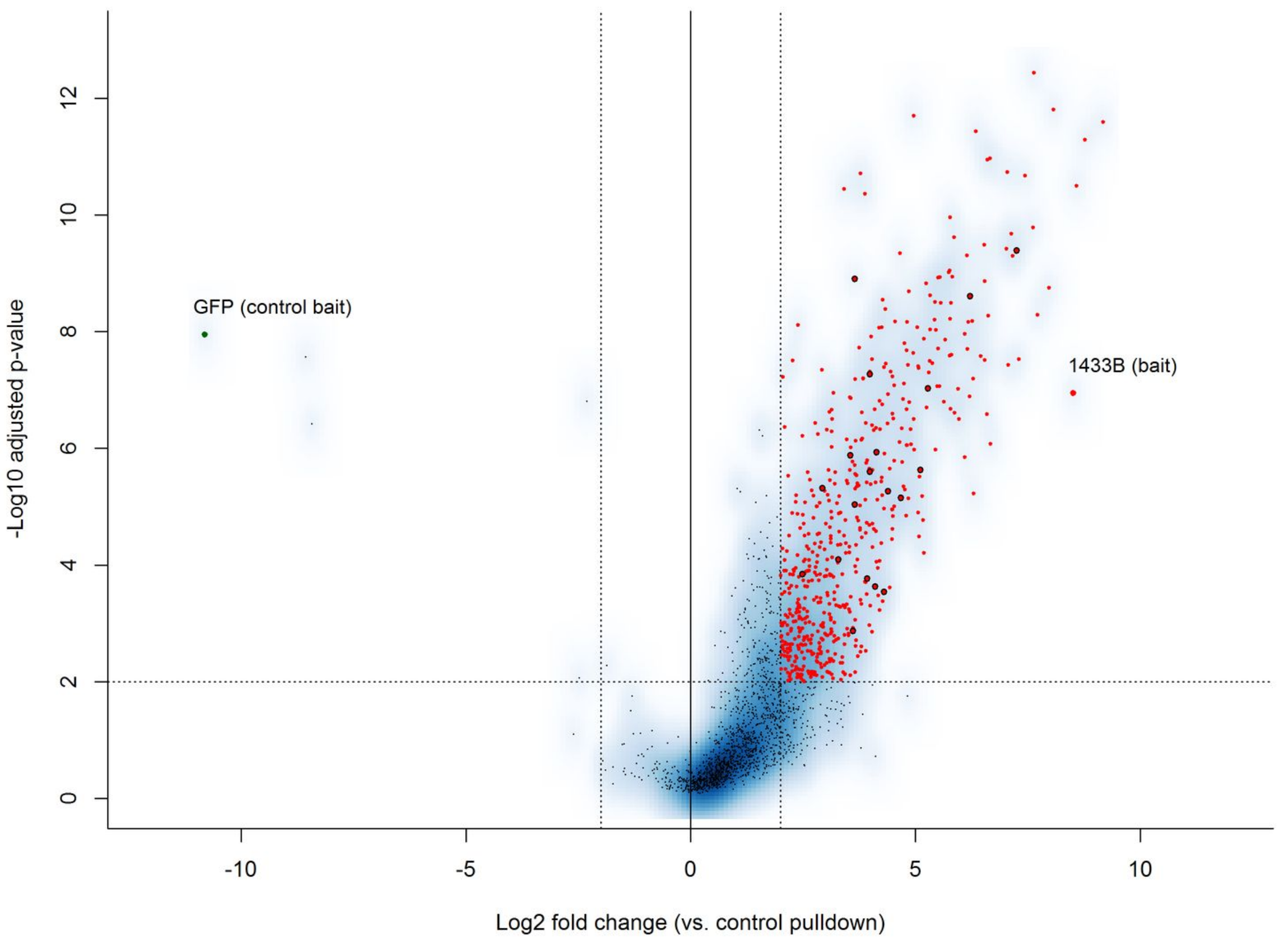




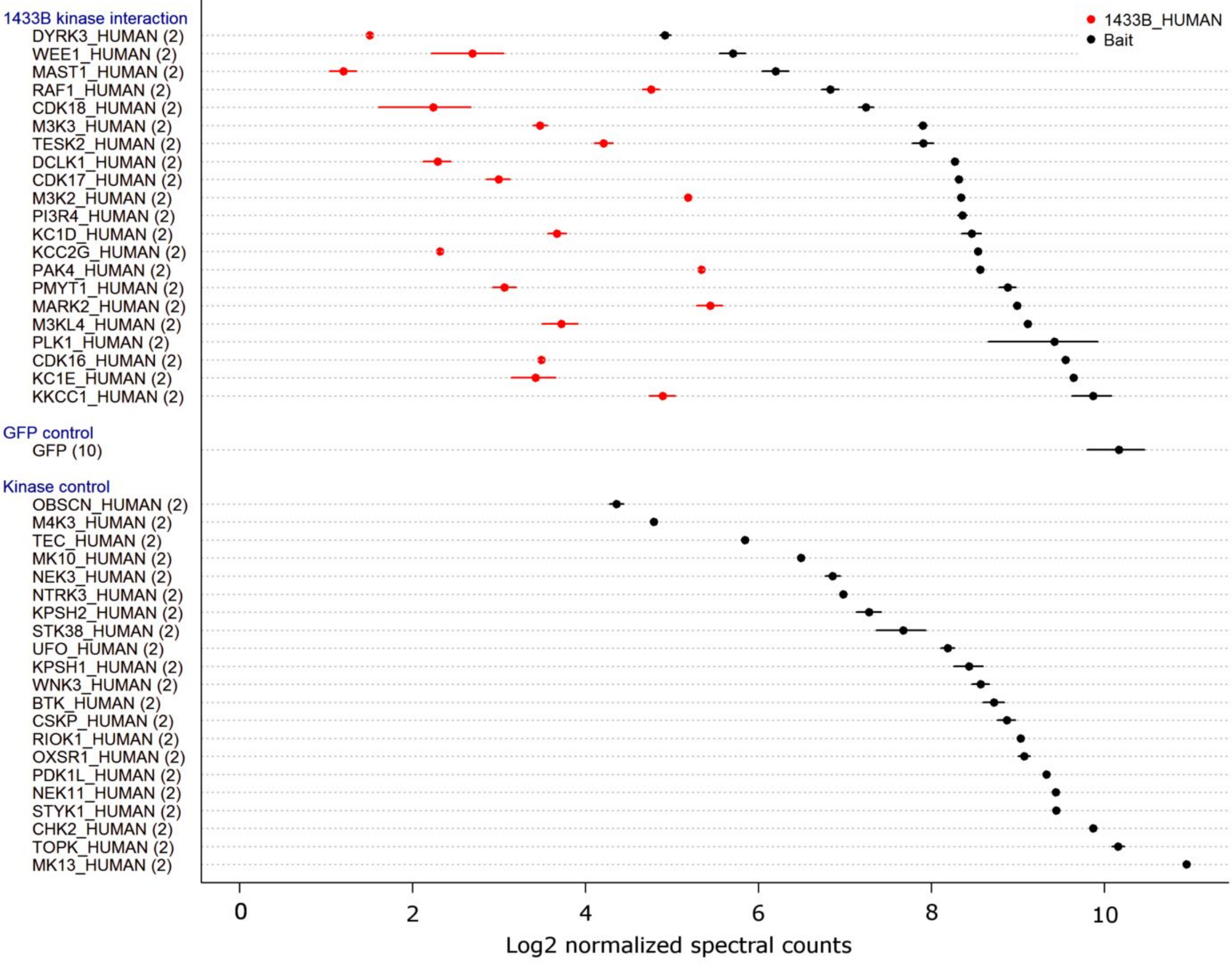




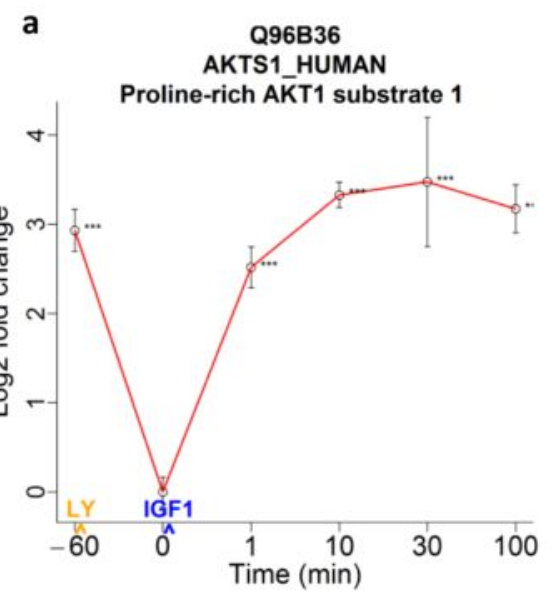

d

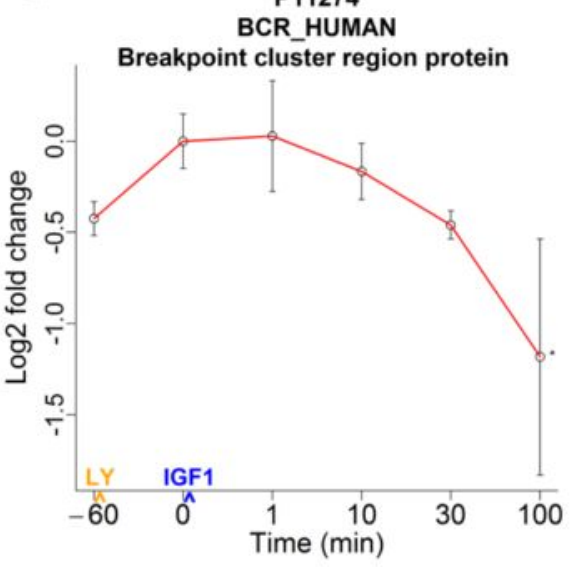

g

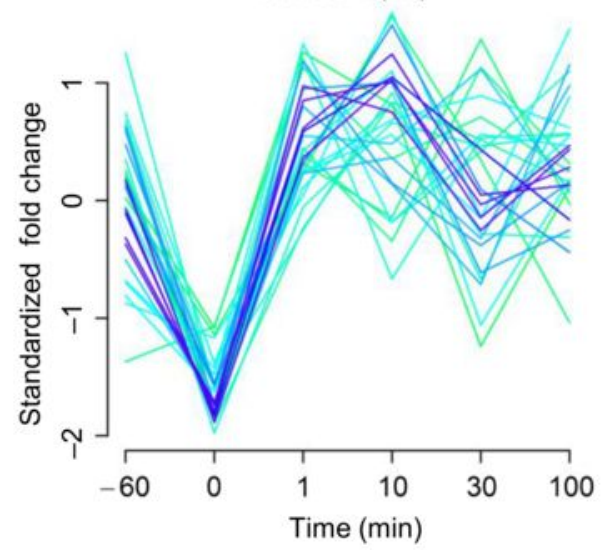

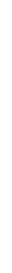

b

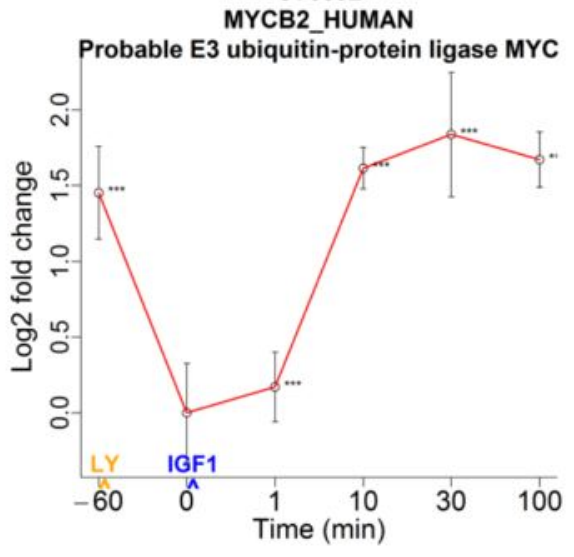

e

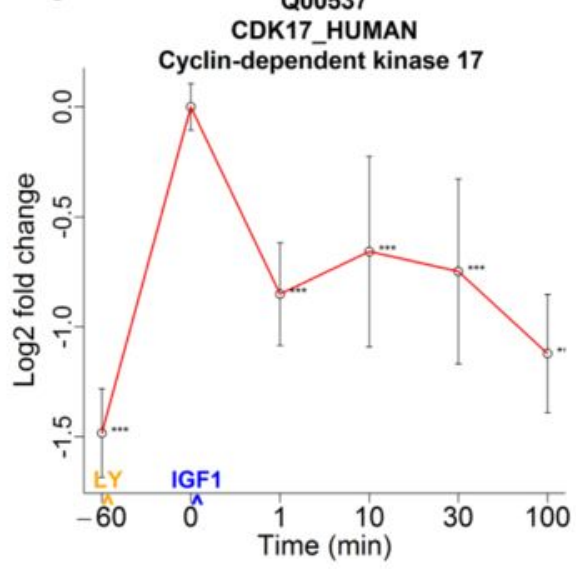

h

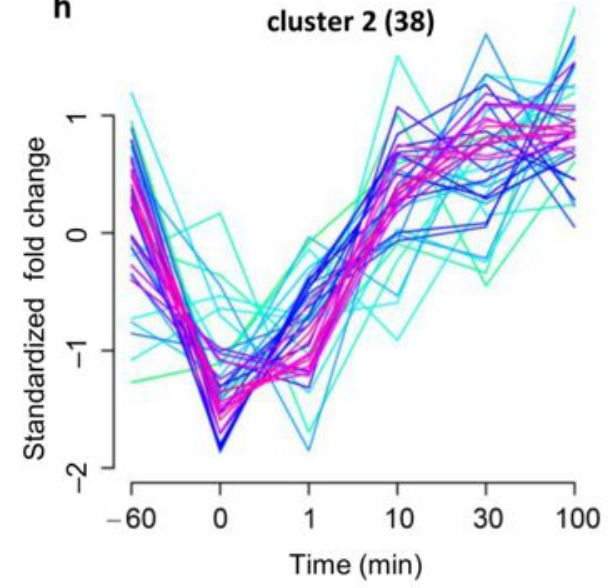

k

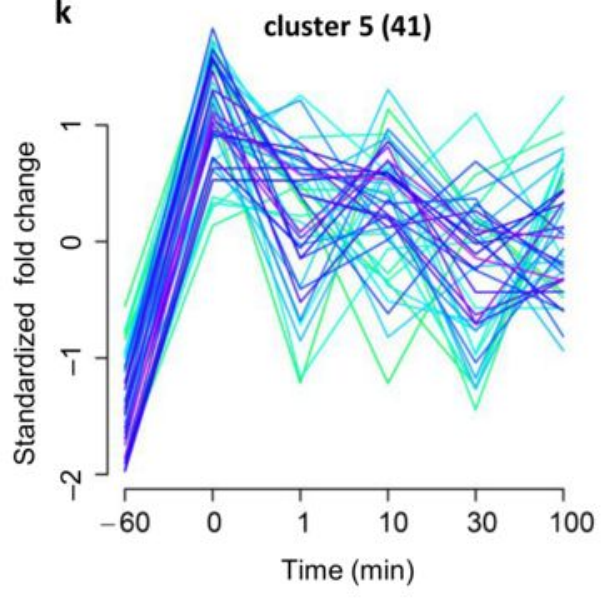

c

Q9UNH5

CC14A HUMAN

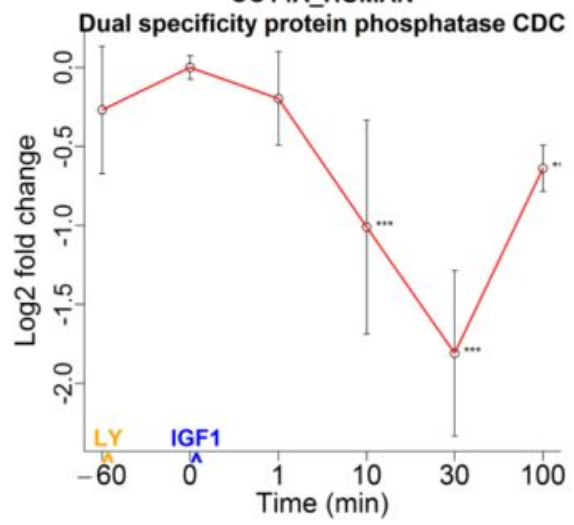

f
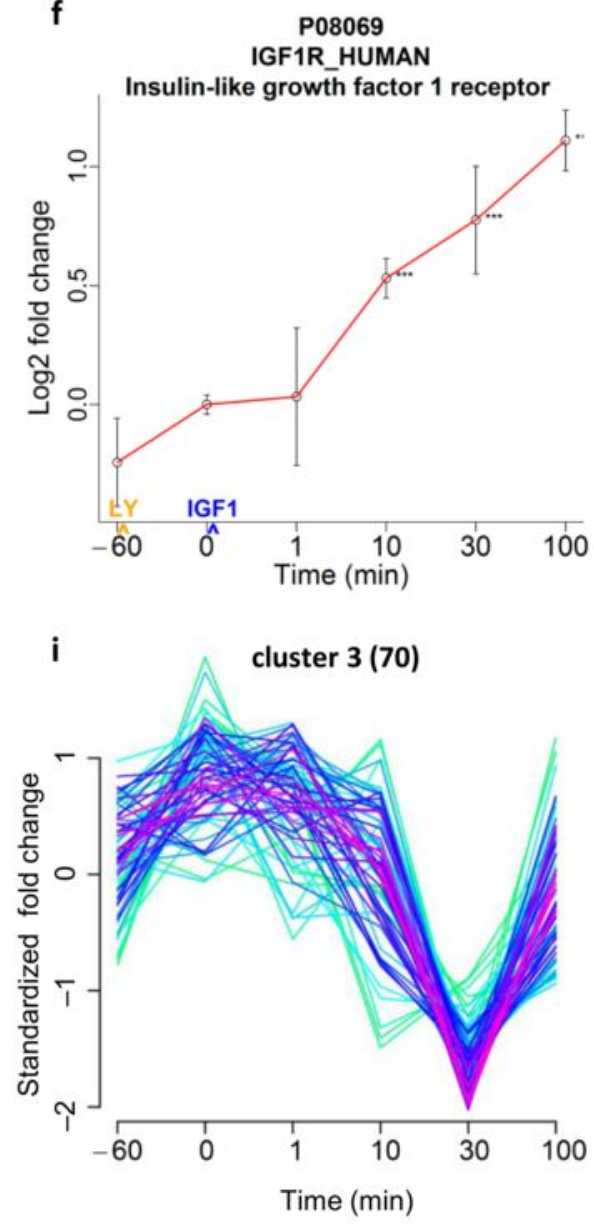

I

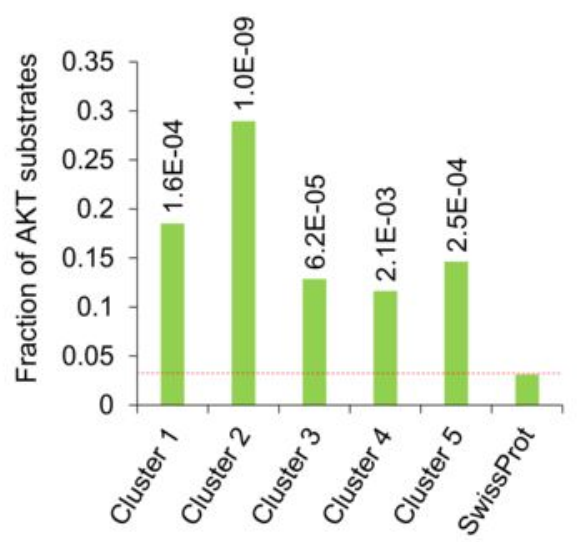


14-3-3 beta interaction (AP-SWATH)

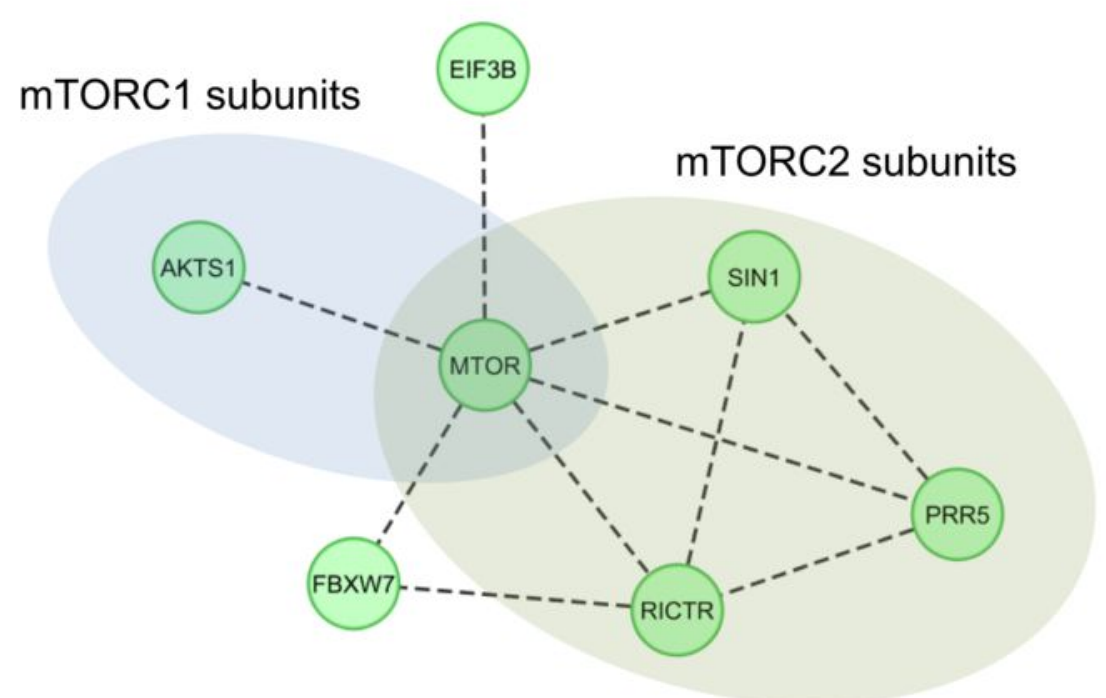

C
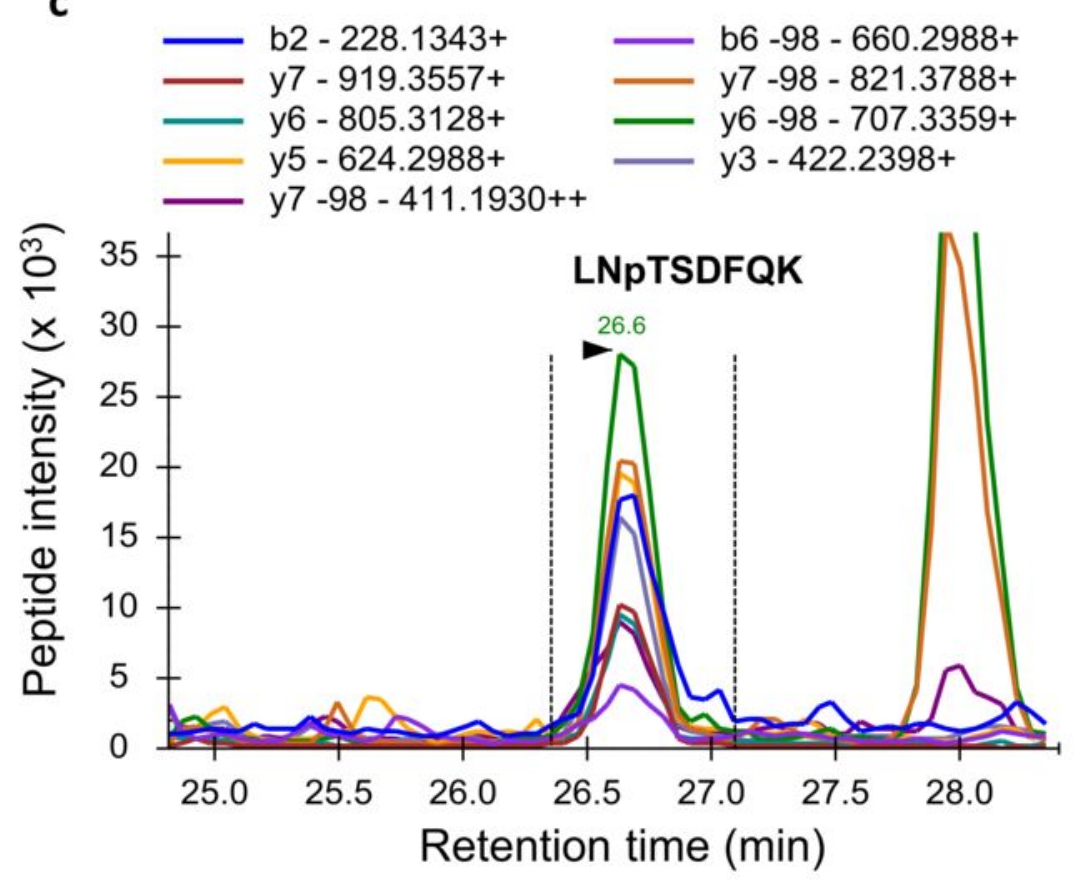

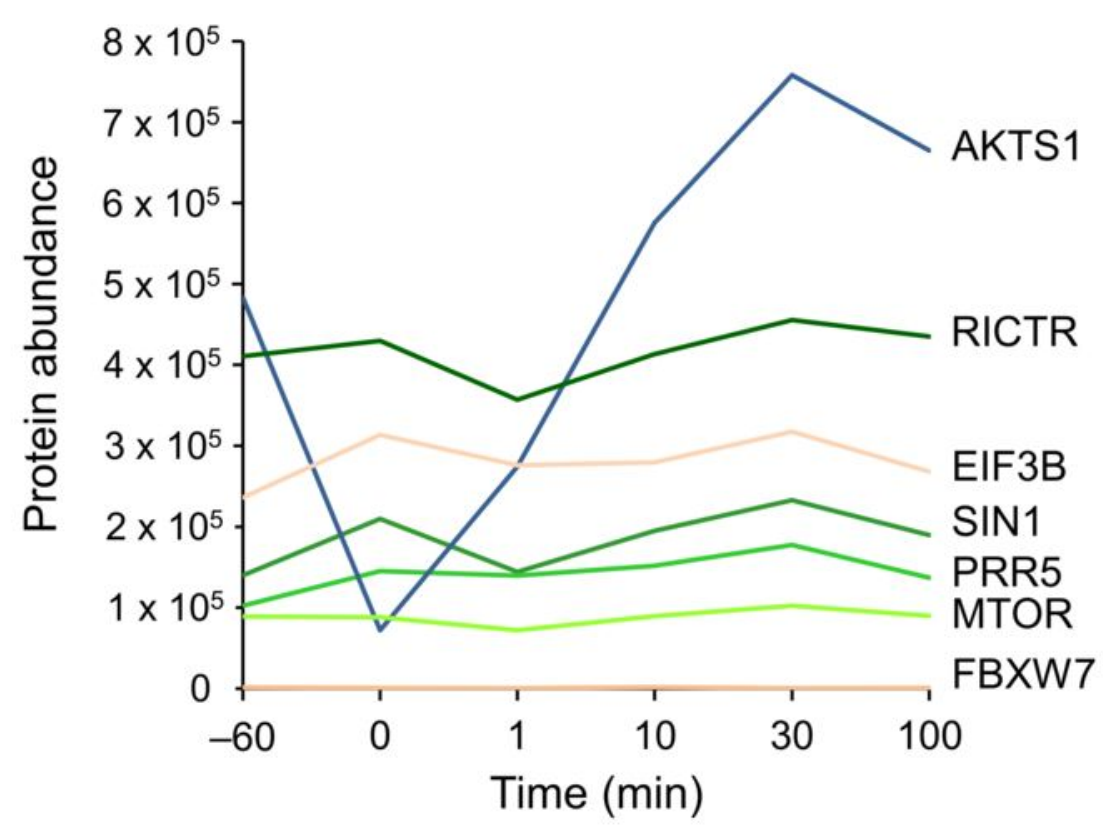

d

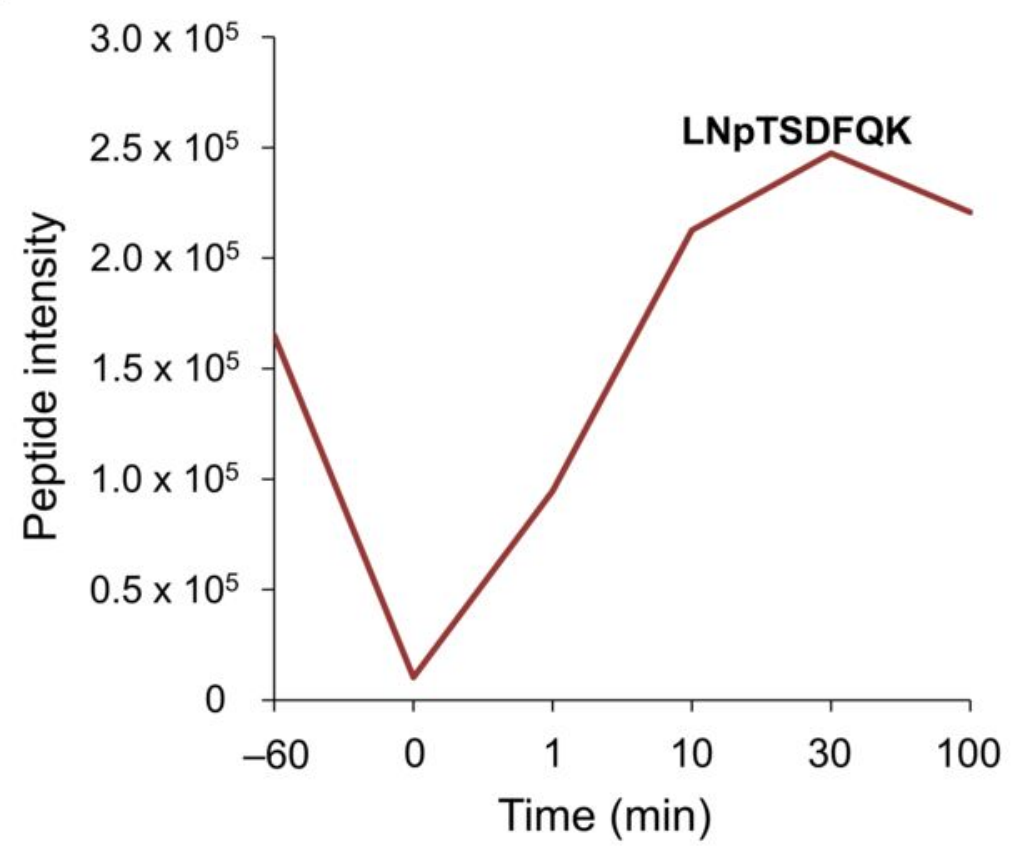


14-3-3 scaffold with phosphorylated 14-3-3 ligand motif

14-3-3 scaffold with unconfirmed attachment site

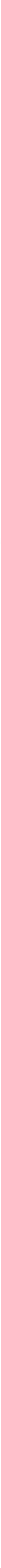




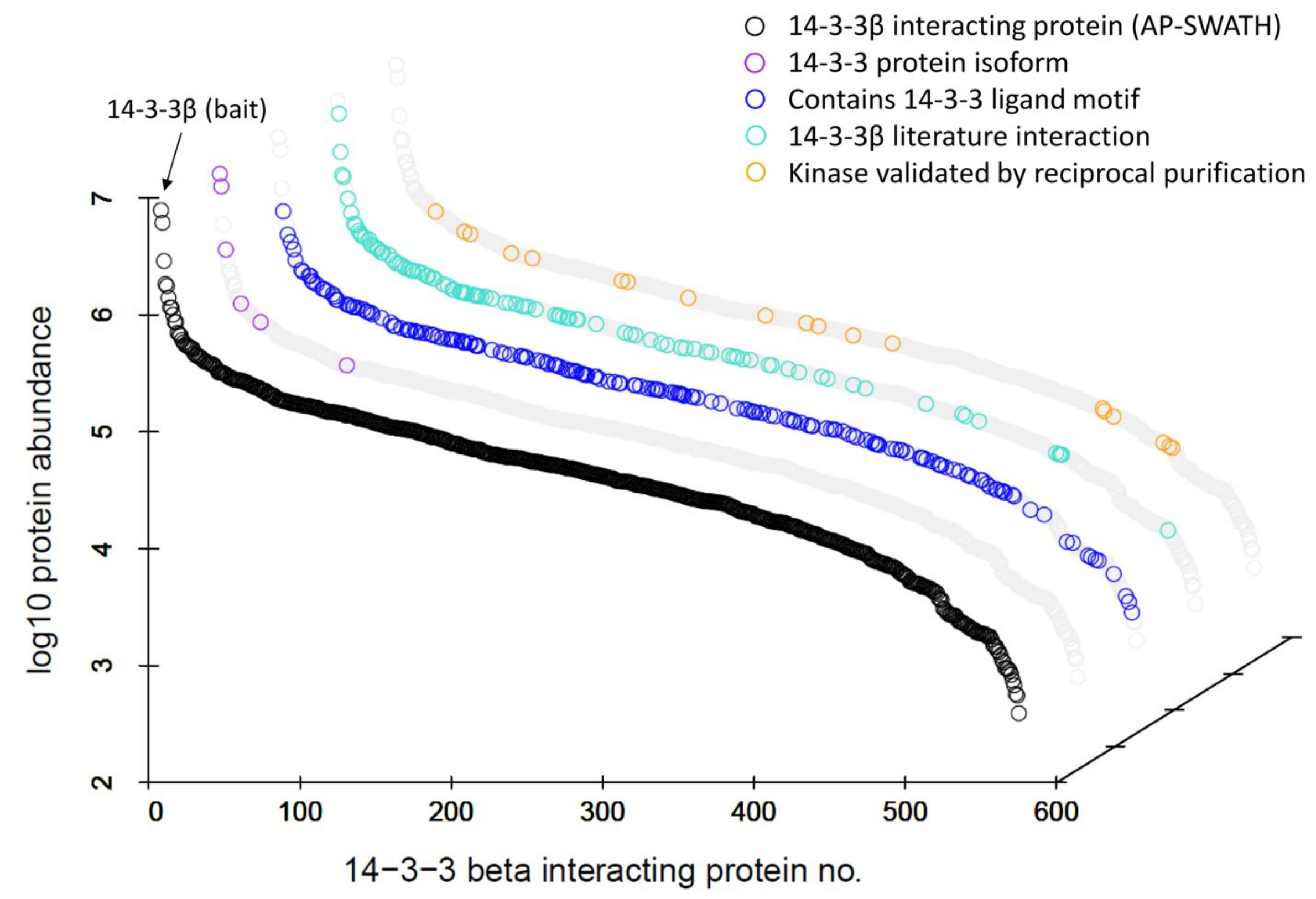

